American Journal of Nursing Science
2020; 9 (4): $276-280$
http://www.sciencepublishinggroup.com/j/ajns
doi: 10.11648 /j.ajns.20200904.29
ISSN: 2328 -5745 (Print); ISSN: $2328-5753$ (Online)

\title{
Construction and Evaluation of the E-clinic Service System in Baoan District, Shenzhen, China
}

\author{
Wei Wang ${ }^{1}$, Weiping Tang ${ }^{1}$, Yali Luo ${ }^{1}$, Naling Zhu ${ }^{1}$, Liling Wang ${ }^{2}$ * \\ ${ }^{1}$ Department of Prevention and Health Care, Shenzhen Baoan Women's and Children's Hospital, Shenzhen, China \\ ${ }^{2}$ Department of Hospital Office, Shenzhen Baoan Women's and Children's Hospital, Shenzhen, China \\ Email address: \\ wangwei-243243@163.com (Wei Wang),644647556@qq.com (Weiping Tang),215543647@qq.com (Yali Luo), \\ 641662556@qq.com (Naling Zhu) \\ ${ }^{*}$ Corresponding author
}

\section{To cite this article:}

Wei Wang, Weiping Tang, Yali Luo, Naling Zhu, Liling Wang. Construction and Evaluation of the E-clinic Service System in Baoan District, Shenzhen, China. American Journal of Nursing Science. Vol. 9, No. 4, 2020, pp. 276-280. doi: 10.11648/j.ajns.20200904.29

Received: June 14, 2020; Accepted: July 3, 2020; Published: July 23, 2020

\begin{abstract}
Objective To construct the regional elimination of mother-to-child transmission (EMTCT) clinic (E-clinic) service system and evaluate its effect. Methods Since November 2018, the E-clinic service system has been established in the obstetric clinic of 18 maternal and child health $(\mathrm{MCH})$ providers in Baoan District, Shenzhen, China, forming a regional service system. In E-clinics obstetricians take care of pregnant women with hepatitis B virus (HBV) infection for complete perinatal services and infectious disease management. Before and after the implementation of the $E$-clinic service system, the changes of HBV screening rate among pregnant women, HBV infected mothers DNA detection rate, mothers with high viral load $\left(>2 \times 10^{6} \mathrm{IU} / \mathrm{mL}\right)$ antiviral therapy (ART) rate and HBV MTCT rate were analyzed. Results From July 2018 to March 2020, there were 162,036 registered pregnant women and 7,358 HBV infected mothers in Baoan District, Shenzhen, including 1,179 mothers with high viral load. The HBV screening rate among pregnant women, HBV infected mothers follow up rate, HBV infected mothers DNA detection rate and ART rate were 97.6\% (124,311/127,379), 99.0\% (5,742/5,801), 82.8\% $(4,805 / 5,801)$ and $70 \%$ (687/982) respectively, which were higher than $95.9 \%(33,247 / 34,657), 93.1 \%(1,449 / 1,557), 49.3 \%$ $(767 / 1,557)$ and $21.3 \%(42 / 197)$ before the implementation of the E-clinic service system. The differences were statistically significant $(P<0.001)$. The HBV MTCT rate decreased from $0.35 \%(12 / 3471)$ to $0 \%(0 / 317)$ after the implementation of the E-clinic service system, and the difference was no statistically significant $(P>0.05)$. Conclusions The E-clinic service system of Baoan District in Shenzhen integrates antenatal care (ANC) and ART, improves the management of HBV infection during pregnancy, further reduces the HBV MTCT, and provides experience for other regions to manage infectious diseases during pregnancy.
\end{abstract}

Keywords: Hepatitis B., Mother-to-Child Transmission, Antiviral Therapy, E-clinic

\section{Introduction}

Hepatitis B remains as the main public health threat in the world, the World Health Organization (WHO) estimated that in 2015, 257 million individuals were living with HBV infection globally, 887,000 people died due to complications of HBV infection [1]. In China, mother-to-child transmission (MTCT) is the major route of HBV transmission, about 90 million people were infected with $\mathrm{HBV}, 6.4 \%$ of women giving birth are living with $\mathrm{HBV}$, among which 1 million HBV infected women give birth each year [2]. Several studies have shown that $90 \%$ of infants infected with $\mathrm{HBV}$ at birth are at risk of chronicity, of which $15 \% \sim 40 \%$ will develop cirrhosis and even liver cancer [3]. MTCT of HBV is still an important issue affecting the health of women and children in China.

Screening of hepatitis B surface antigen (HBsAg) for pregnant women, timely using of hepatitis $\mathrm{B}$ vaccine (HepB) and extra hepatitis B immunoglobulin (HBIG) to infants whose mothers are HBsAg positive, and combination another two doses of HepB are the main measures to prevent MTCT of $\mathrm{HBV}$ in China. At the same time, it is recommended to carry out HBV DNA detection in the areas where conditions permit, 
and if necessary, to refer to the infectious disease specialist for treatment [4]. At present, HBV infected patients in China are mainly referred to the infectious disease hospital or the Department of infection and Hepatology for antiviral therapy (ART). Pregnant women with HBV infection needed referral across different health facilities and reduced the adherence of ART. Meanwhile, the lack of unified management of HBV infected pregnant women in health facilities makes the continuous and integrity of treatment difficulty. Previously, the United States, Europe, and many regions guidelines for the management of hepatitis B recommended the use of antiviral drugs for pregnant women with high viral load in the second or third trimester [5-7]. In 2017, we have developed a Management Algorithm for Interrupting MTCT of HBV, it recommended that among pregnant women with $\mathrm{HBV}$ DNA $>2 \times 10^{6} \mathrm{IU} / \mathrm{mL}$, ART with either tenofovir disoproxil fumarate (TDF) or telbivudine (LdT) should be administered [8]. For further reducing the risk of HBV MTCT, the China's Guidelines for the Prevention and Treatment of Mother-to-child Transmission of Hepatitis B virus (2019 edition) [9] recommended that for pregnant women with HBV DNA $>2 \times 10^{5} \mathrm{IU} / \mathrm{mL}, \mathrm{TDF}$ and LdT could be administered during pregnancy.

Baoan as the most populous district in Shenzhen has a huge number of migrants from other parts of China, who on average account for $90 \%$ of the whole population living in Baoan District. The total population in this district was estimated to be 5.5 million and the prevalence of $\mathrm{HBsAg}$ in pregnant women was $9 \%$ [10]. There are 18 maternal and child health $(\mathrm{MCH})$ providers in Baoan District. In 2018 approximately $8,000 \mathrm{HBV}$ infected pregnant women had their pregnancy registration here and the number of newborns reached 4,000. In 2017 Baoan District was officially appointed as a participant for the elimination of mother-to-child transmission (EMTCT) pilot program launched by National Health Commission. In 2018 Baoan District joined the Guangdong province's campaign of Zero Transmission of MTCT for HBV and align all the EMTCT work in an integrated approach. How to improve the adherence of HBV infected pregnant women, reduce unnecessary referral of patient across different health facilities, and achieve the goal of the pilot area as soon as possible, are our urgent challenges to be promoted.

According to the above challenges, a brand new service model called EMTCT clinic (E-clinic) was launched in the pilot area in November 2018. It was put in use for integration of antenatal care (ANC) with infection management. In E-clinics obstetricians with competency of infectious disease management take care of a pregnant woman with HBV infection for complete perinatal services. By doing this we have managed the infectious diseases during pregnancy and EMTCT of HBV.

\section{Methods}

\subsection{Testing Triage and Color Management}

We have taken an innovative approach in arranging free screening of HIV, syphilis and HBV before registration procedure for ANC visits to increase the coverage. This has resulted in reducing the number of missed cases. Besides, HBsAg positive pregnant women shall be managed by specially-assigned persons according to high risk pregnancy classification purple and triage to E-clinic. Since September 2018, $18 \mathrm{MCH}$ providers have provided the first HBV DNA detection free for HBsAg positive pregnant women.

\subsection{Initial Assessment and Consultation}

The obstetricians in the E-clinic assessed and educated the HBV infected pregnant women, popularized the knowledge related to hepatitis $\mathrm{B}$, and provided relevant consultation services such as reasonable mode of delivery, discontinuation of antivirals, breastfeeding and neonatal immunoprophylaxis.

\subsection{Antiviral Intervention During Pregnancy}

According to the China' Management Algorithm for Interrupting MTCT of HBV [8], if HBV DNA level is greater than $2 \times 10^{6} \mathrm{IU} / \mathrm{mL}$ or alanine aminotransferase (ALT) level abnormal, ART with either TDF or LdT should be administered after patient education and informed consent at gestational weeks 24 to 28 .

\subsection{Follow up Evaluation of Mothers and Infants}

Since July 2018, mothers with HBsAg positive after delivery should be followed up. Infants born to HBsAg positive mothers since June 2017 should be followed up for completion of hepatitis $\mathrm{B}$ vaccination and post vaccination serologic testing (PVST) of 7 15 months old.

\subsection{Information Management}

Strengthen information management and utilization. The real-time monitoring of lab with $\mathrm{HBsAg}$ positive results will automatically send messages to the ANC pregnant women, remind them to make an appointment with the E-clinic. For pregnant women with high viral load, individual case follow up were arranged or the lab information system shall set up a message to remind them to ART at gestational weeks 24 to 28 .

\subsection{Stigma and Discrimination Reduction}

Among all participating health facilities, we provided training workshops to increase the awareness of privacy protection, informed consent and discrimination reduction. We also set up a specific communication group for counseling and reducing any type of stigma and discrimination in health facilities they experienced.

\subsection{Data Resources}

$18 \mathrm{MCH}$ providers and community health centers reported monthly statements to the EMTCT pilot project office, which mainly included the screening report form for pregnant women, the follow up case form for mothers infected with $\mathrm{HBV}$ and the infant exposed to HBV, the EMTCT pilot 
project office collated and summarized.

\subsection{Data Analysis}

Excel 2013 software was used for data collation and verification, and SPSS 20.0 software was used for data statistical analysis. Compare the changes of HBV screening rate among pregnant woman, HBV infected mothers DNA detection rate, mothers with high $\mathrm{HBV}$ virus load $\left(>2 \times 10^{6}\right.$ $\mathrm{IU} / \mathrm{mL}$ ) ART rate and $\mathrm{HBV}$ MTCT rate before the implementation of the E-clinic service system (July 2018 to October 2018) and after the implementation (November 2018 to March 2020). The changes of HBV MTCT rate between June 2017 to October 2018 and November 2018 to December 2018 were compared. The Chi-square test was used to analyze the rate, and $P<0.05$ was considered statistically significant.

\section{Results}

\subsection{Comparison of Main Management Indicators}

From July 2018 to March 2020, there were 162,036 women had their pregnancy registration in Baoan District, Shenzhen, and 7,358 cases of HBV infected mothers, among which 1,179 cases were of high viral load. the HBV screening rate among pregnant women, HBV infected mothers follow up rate, HBV infected mothers DNA detection rate and ART rate were $97.6 \%$ (124311/127379), 99.0\% (5742/5801), 82.8\% (4805/5801) and $70 \%$ (687/982), respectively, all of them counterparts were higher than $95.9 \%$ (33247/34657), 93.1\% (1449/1557), 49.3\% (767/1557) and $21.3 \%$ (42/197) before the implementation of the $E$-clinic service system. All the differences were statistically significant $(P<0.001)$. Table 1.

Table 1. Comparison of main management indicators of the E-clinic service system.

\begin{tabular}{|c|c|c|c|c|c|c|}
\hline Indicators & & Before E-clinic n (\%) & After E-clinic n (\%) & Total n (\%) & $x^{2}$ & $\mathbf{P}$ \\
\hline \multirow{3}{*}{$\begin{array}{l}\text { Pregnant women } \\
\text { HBV screening }\end{array}$} & Yes & 33247 (95.9) & $124311(97.6)$ & $157558(97.2)$ & \multirow{3}{*}{279.34} & \multirow{3}{*}{$<0.001$} \\
\hline & No & $1410(4.1)$ & $3068(2.4)$ & $4478(2.8)$ & & \\
\hline & Total & $34657(21.4)$ & $127379(78.6)$ & $162036(100.0)$ & & \\
\hline \multirow{3}{*}{$\begin{array}{l}\text { HBV infected mothers } \\
\text { DNA detection }\end{array}$} & Yes & $767(49.3)$ & $4805(82.8)$ & $5572(75.7)$ & \multirow{3}{*}{752.56} & \multirow{3}{*}{$<0.001$} \\
\hline & No & $790(50.7)$ & $996(17.2)$ & $1786(24.3)$ & & \\
\hline & Total & $1557(21.2)$ & $5801(78.8)$ & $7358(100)$ & & \\
\hline \multirow{3}{*}{$\begin{array}{l}\text { HBV infected mothers } \\
\text { ART }^{*}\end{array}$} & Yes & $42(21.3)$ & $687(70.0)$ & $729(61.8)$ & \multirow{3}{*}{164.49} & \multirow{3}{*}{$<0.001$} \\
\hline & No & $155(78.7)$ & $295(30.0)$ & $450(38.2)$ & & \\
\hline & Total & $197(16.7)$ & $982(83.3)$ & $1179(100)$ & & \\
\hline \multirow{3}{*}{$\begin{array}{l}\text { HBV infected mothers } \\
\text { follow up }\end{array}$} & Yes & $1449(93.1)$ & $5742(99.0)$ & 7191 (97.7) & \multirow{3}{*}{193.91} & \multirow{3}{*}{$<0.001$} \\
\hline & No & $108(6.9)$ & $59(1.0)$ & $167(2.3)$ & & \\
\hline & Total & 1557 (21.2) & $5801(78.8)$ & $7358(100.0)$ & & \\
\hline
\end{tabular}

*ART: antiviral therapy.

\subsection{The Trend of DNA Detection Rate and ART Rate in HBV Infected Mothers}

Since the implementation of the free HBV DNA detection in September 2018, the DNA detection rate in HBV infected mothers has continued to rise, and in the first quarter of 2020, it was more than $90 \%$. Since the implementation of the E-clinic service system in November 2018, the ART rate in HBV infected mothers has continued to increase, nearly $80 \%$. Figure 1 .

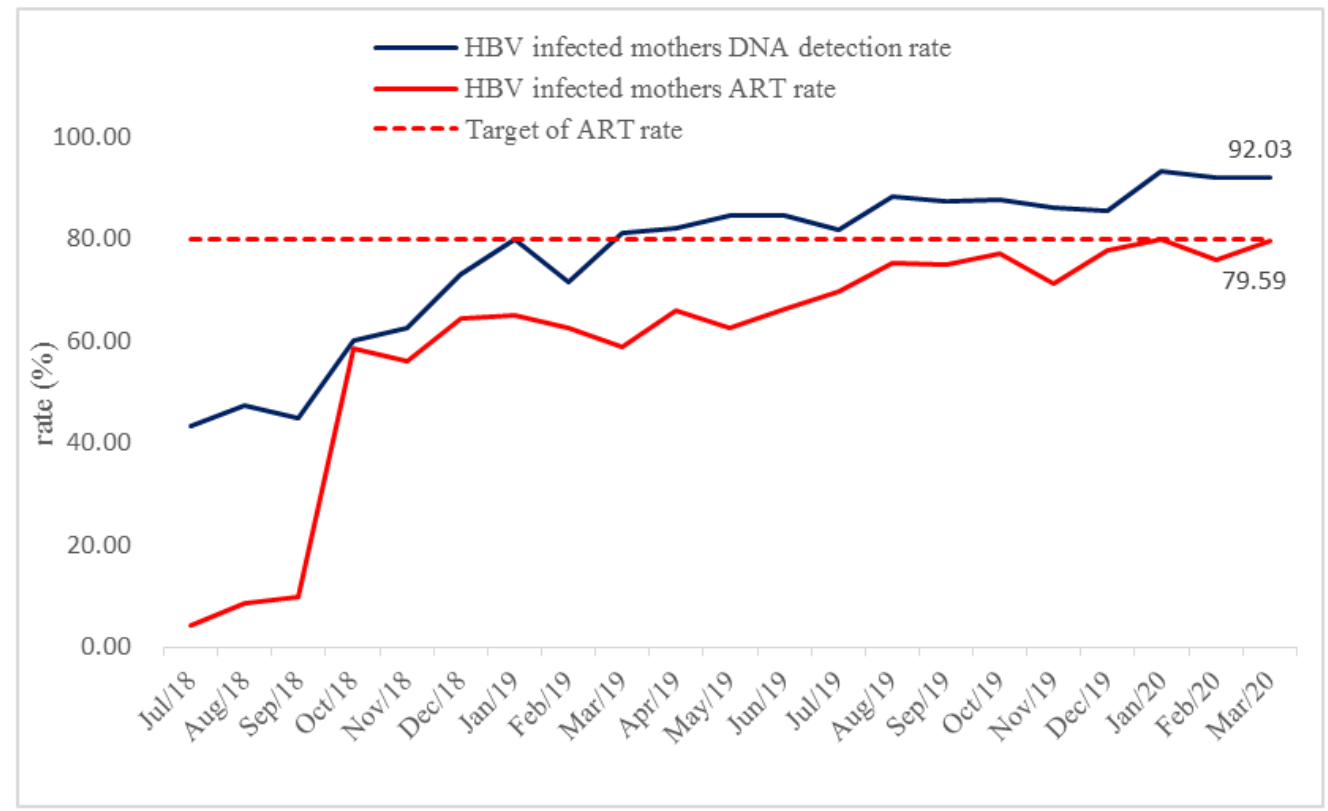

ART: antiviral therapy.

Figure 1. DNA detection rate and ART rate of HBV infected mothers in Baoan District, Shenzhen from July 2018 to March 2020. 


\subsection{Decrease of HBV MTCT Rate}

From July 2017 to December 2018, a total of 7,309 infants exposed to HBV, of which 6,167 children aged 7 15 months old were followed up by $\mathrm{MCH}$ providers and community health centers in Baoan District, with a follow up rate of $84.4 \%$, including 3,788 children for PVST, with a PVST covering $61.4 \%$. The HBV MTCT rate was $0.32 \%(12 / 3788)$ in Baoan District, Shenzhen, and the HBV MTCT rate was $0.35 \%(12 / 3471)$ and $0 \%(0 / 317)$ before and after the E-clinic service system implementation, respectively, with no statistically significant difference $(P>0.05)$.

\section{Discussion}

\subsection{Background of the E-clinic Service System Construction}

Baoan District in Shenzhen has a huge number of migrants from other parts of China, and the prevalence of HBsAg in pregnant women is $9 \%[10]$, which belongs to the high HBV prevalence area (according to the definition of WHO, the area with $\mathrm{HBsAg}$ positive rate $>8 \%$ is the high $\mathrm{HBV}$ prevalence area) [11]. In the past, all HBV infected pregnant women were referred to multiple health facilities for treatment, and there is no unified management of pregnant women with HBV infection. Based on this, the EMTCT pilot project office promoted $18 \mathrm{MCH}$ providers to establish the E-clinic service system, integrated ANC and management infectious diseases, provided one-stop services, improved maternal compliance, and EMTCT of HBV.

\subsection{The Effect of the E-clinic Service System}

China's Integrated Prevention of MTCT of HIV, Syphilis, and HBV program (iPMTCT program) was launched in 2010, 2015 edition of the Protocol for iPMTCT program required all pregnant women to screen HBsAg. In 2017, the national coverage of HBsAg screening test among pregnant women exceeded 99.5\% [12]. We promoted screening first of HIV, syphilis and HBV to increase the coverage, this has resulted in an expanded coverage of universal screening from $95.9 \%$ to 97.6\%. Measures to EMTCT of HBV were effectively implemented. Annually a large number of people frequently move in and out of Baoan District, some untested pregnant women have been screened in other areas, but there are still cases refusing to be tested. For those who refuse to be tested, it is suggested that further education should be strengthened, early and timely screening can prolong the intervention time for those who are infected.

Although the HBsAg screening coverage among pregnant women has reached $99.5 \%$, the national coverage of DNA detection and ART among pregnant women were still less than $30 \%$, and the remote, rural areas were lower than that of the national level because of limited health resources and public health education for HBV [2]. Several studies suggested that antiviral treatment during the third trimester is effective in decreasing the risk of MTCT among infants born to mothers with high viral load $[13,14]$. Since the implementation of the E-clinic, Baoan District has initialled pregnancy intervention according to Management Algorithm for Interrupting MTCT of HBV, and the ability of DNA detection and ART in HBV infected mothers have been significantly improved, with the HBV DNA detection rate and ART rate were $82.8 \%$ and $70 \%$, respectively. In the first quarter of 2020, the HBV DNA detection rate was above $90 \%$ and the ART rate close to $80 \%$, indicating that the E-clinic service system has made considerable progress.

A total of 3,788 infants exposed to HBV for PVST, HBV MTCT rate was $0.32 \%, 12$ cases were infected with HBV, reaching the national goal of EMTCT $(<2 \%)$. After the implementation of the E-clinic service system, there was no case of infection. Although the HBV MTCT rate decreased, but no statistical difference, suggesting that more samples were needed and time to observe.

\subsection{Advantages and Features of the E-clinic Service System}

The E-clinic is put in use for integration of ANC with infection management, which promotes the homogeneity of services in Baoan District. Pregnant women with HBV infection can get services of ANC and ART in the E-clinic, it also reduced the invisible stigma and discrimination between health facilities. Such a change reduced unnecessary referral of patient across different disciplines and increased the adherence of ART. The one-stop service provided by the E-clinic has effectively strengthened the management of infectious diseases during pregnancy and EMTCT of HBV. Meanwhile, it is beneficial to the construction and development of the subspecialty of obstetrics, and provides reference experience for exploring the management of infectious diseases in obstetric system.

\subsection{Challenges and Next Steps}

Though we have established the E-clinic service system for management infectious diseases at $18 \mathrm{MCH}$ providers in Baoan District, based on previous work, there are still some challenges in the E-clinic mode of HBV EMTCT. There are differences in the level and management of medical service between public and private facilities, and the service quality is difficult to keep the same. Limited by professional ability, the E-clinic has a weak ability to treat severe liver diseases such as acute hepatitis and cirrhosis, such pregnant women still need to be referred to the infectious disease hospital. Pregnant women who use antiviral drugs are prone to rebound and increase of viral load after discontinuation of drug delivery, and the effect of discontinuation on drug resistance is unknown. Controversies exist on the use of ART to EMTCT of HBV [15]. The WHO currently does not officially recommend the routine use of ART to prevent MTCT of HBV because of the lack of sufficient evidence supporting its implementation [16]. For children with HBV infection, there is no guidelines for treatment management. Based on the above problems, the next step will be to strengthen the training 
of obstetricians, requiring them to master the Management Algorithm for Interrupting MTCT of HBV. Enhance the cooperation and exchange between public and private facilities. For pregnant women who receive ART, continuous postpartum monitoring will be needed, and abnormal cases timely referral. Strengthen the management of children infected through MTCT of HBV and follow up the progress of the disease.

\section{Conclusion}

Baoan District of Shenzhen has successfully established and implemented the E-clinic service system in $18 \mathrm{MCH}$ providers, which has expanded coverage of screening, HBV DNA detection and ART among HBV infected pregnant women, also, one-stop service is helpful for medical institutions to manage $\mathrm{HBV}$ infected pregnant women and improve their compliance. It reduces the MTCT rate of HBV and provides reference for other areas to EMTCT.

\section{Acknowledgements}

The authors are particularly thankful to obstetricians in E-clinic, from $18 \mathrm{MCH}$ providers in Baoan District, Shenzhen, China. This study received no supports from any funding and the authors declared no conflict of interest.

\section{References}

[1] ZOU H., CHEN Y., DUAN Z., ZHANG H., PAN C. Virologic factors associated with failure to passive-active immunoprophylaxis in infants born to $\mathrm{HBs} A g$-positive mothers. J. VIRAL HEPATITIS. 2012; 19: e18-25.

[2] LIANG X., BI S., YANG W., et al. Epidemiological serosurvey of hepatitis B in China--declining HBV prevalence due to hepatitis B. vaccination. VACCINE. 2009; 27: 6550-57.

[3] WANG FS, FAN JG, ZHANG Z., GAO B., WANG HY. The global burden of liver disease: the major impact of China. HEPATOLOGY. 2014; 60: 2099-108.

[4] Department of maternal and child health services, national health and Family Planning Commission, Center for Maternal and Child Health, CDC. Interpretation of the Implementation Plan for the Prevention of Mother-to-child Transmission of HIV, Syphilis and HBV (2015 edition) Chinese Journal of Women and Children Health. (Zhonghua Fu You Wei Sheng Za Zhi) 2015; 6 (6): 1-2. (in Chinese).
[5] TERRAULT NA, LOK A., MCMAHON BJ, et al. Update on prevention, diagnosis, and treatment of chronic hepatitis $\mathrm{B}$ : AASLD 2018 hepatitis B guidance. HEPATOLOGY. 2018; 67: 1560-99.

[6] SARIN SK, KUMAR M., LAU GK, et al. Asian-Pacific clinical practice guidelines on the management of hepatitis B.: a 2015 update. HEPATOL INT. 2016; 10: 1-98.

[7] EASL 2017 Clinical Practice Guidelines on the management of hepatitis B virus infection. J HEPATOL. 2017; 67: 370-98.

[8] HOU J., CUI F, DING Y., et al. Management Algorithm for Interrupting Mother-to-Child Transmission of Hepatitis B. Virus. CLIN GASTROENTEROL H. 2019; 17: 1929-36.

[9] YINGREN Z., GUIQIANG W., WENHONG Z., DISEASES CSOI. Chinese Guidelines for prevention and Treatment of Mother-to-child Transmission of hepatitis B. Virus (2019 edition).

[10] Chin J. Infect Dis (Zhonghua Chuan Ran Bing Za Zhi) 2019; 7 (37): 388-396. (in Chinese).

[11] TAOYANG C., YAN J., YU Z., et al. Mutations in hepatitis B. virus genome involved inimmunoprophylaxis failure against vertical transmission. Chin J. Microbiol Immunol. (Zhonghua Wei Sheng Wu He Mian Yi Xue Za Zhi) 2009; 6 (29): 538-543. (in Chinese).

[12] OZARAS R., INANC BI, YEMISEN M., TABAK F. Epidemiology of HBV subgenotypes D. Clin Res Hepatol Gastroenterol. 2015; 39: 28-37.

[13] WANG A., QIAO Y., DOU L. Challenges of eliminating mother-to-child transmission of HIV, syphilis and hepatitis B in China: a cross-sectional survey. Lancet. 2018; 392.

[14] HAN GR, CAO MK, ZHAO W., et al. A. prospective sand open-label study for the efficacy and safety of telbivudine in pregnancy for the prevention of perinatal transmission of hepatitis B virus infection. J. HEPATOL. 2011; 55: 1215-21.

[15] ZHANG H., PAN CQ, PANG Q., TIAN R., YAN M., LIU X. Telbivudine or lamivudine use in late pregnancy safely reduces perinatal transmission of hepatitis B virus in real-life practice. HEPATOLOGY. 2014.

[16] XUERU Y., ZHIHUA L., ZHIHONG L., et al. Getting to zero mother-to-child transmission of hepatitis $B$. virus: dream and challenge. Chin J. Hepatol (Zhonghua Gan Zang Bing Za Zhi) 2018; (4): 262-265. (in Chinese).

[17] Guidelines for the Prevention, Care and Treatment of Persons with Chronic Hepatitis B Infection. Geneva: World Health Organization, 2015. 